\title{
An Application of Random Fixed Point Theorem in Integral Equation
}

\author{
Mukti Gangopadhyay ${ }^{1}$, Pritha $\operatorname{Dan}^{2}$ and M. Saha ${ }^{3}$ \\ ${ }^{1}$ Panihati Mahavidyalaya, Barasat Road, Kolkata-700 110, West Bengal. India. \\ E-mail: muktigangopadhyay@yahoo.com \\ ${ }^{2}$ Department of Mathematics, University Institute of Technology, The University of Burdwan, \\ Burdwan-713104, West Bengal, India. \\ E-mail:danpritha@gmail.com \\ ${ }^{3}$ Department of Mathematics, The University of Burdwan, Burdwan-713 104, West Bengal,India. \\ E-mail:mantusaha@yahoo.com
}

Abstract: In this paper random fixed point theorem has been applied seeking solution of Volterra type integral equation involving more generous kernel.

2000 Mathematics Subject Classifications: Primary 47H10; Secondary 54H25, $60 H 25$

Key words and Phrases: random fixed point, Caratheodory function, random Volterra integral equation

\section{Introduction:}

Currently theories of random equations have attracted many researchers. Various mathematical processes in physical, biological and engineering sciences have given rise to random/stochastic integral equation. Typical mathematical treatments in such problems may be found in recent works of Bharucha-Reid [3] and Tosokos [4] where one sees that fixed point theory has been extensively employed in this connection. In general, random analogues of deterministic results in fixed point theory have also been objects of many investigations. For example, works of Ding Xieping and Wang Fan [5], Anderson [8], Padgett and Tsokos [14], Lee and Padgett [2] bear this point.

In 1972, a book 'Random Integral Equations' authored by A.T. Bharucha -Reid had given much impetus in study of random fixed point theory. So one finds some important contributions of eminent workers like S.Itoh [13] and Ding Xieping [5], Saha and Debnath [9], Saha, Gangopadhyay and Debnath [10]. Operator involved in above investigations enjoy some kind of contractive character either in itself or in some of its iterates.

Another milestone of study of random equations had been noted in deliberations of Prague school of probabilists under directions of A. Spacek[1], and works of R. Subramanium and K. Balachandram [11] are worth referring to, as they had been responsible to establish existence criterion of solution of a very wide class of stochastic integral equations of volterra types.

Let $(X,\|\| \|)$ be a separable Banach space and $(\Omega . / \mu)$ be a complete $\sigma$-finite measure space. A mapping $x: \Omega \rightarrow X$ is said to be measurable if for any closed subset $B$ of $X, X^{-1}(B)=\{\omega: x(\omega) \in B\} \in \mathscr{d}$.

Let $I=\left[t_{0}, t_{0}+a\right]\left(t_{0} \geq 0, a>0\right)$ be a closed interval of the real line $\square$. We say that $u: \Omega \times I \rightarrow X$ is a caratheodory function (see [6]) if for each $t \in I, u([, t)$ is measurable and for each $\omega \in \Omega, u(\omega, \square)$ is continuous.

Analogously, $\quad k: \Omega \times I \times I \times X \rightarrow X \quad$ is a caratheodory function if for each $(t, s, x) \in I \times I \times X, k(\square, t, s, x)$ is measurable and for each $\omega \in \Omega, k(\omega, \square, \square)$ is continuous.

Let $C(I, X)=\{x: I \rightarrow X: x$ is continuous $\}$ and

$$
\|x\|_{I}=\max _{t \in I}\|x(t)\| \text {. }
$$


Then $\left(C(I, X),\|X\|_{I}\right)$ is a separable Banach space. Let $C(\Omega \times I, X)$ denote the set of all caratheodory functions $u: \Omega \times I \rightarrow X$ and $C(\Omega \times I \times I \times X, X)$ denote the set of all caratheodory functions $k: \Omega \times I \times I \times X \rightarrow X$.

Lemma 2.1 [6]: A function $u \in C(\Omega \times I, X)$ if and only if the function $\omega \rightarrow u(\omega, t)$ as a function from $\Omega$ into $C(I, X)$ is measurable. i.e., $u(\square, t)$ is a $C(I, X)$-valued measurable function.

Lemma 2.2 [6]: Let $T: \Omega \times X \rightarrow X$ be a charatheodory function and let $x: \Omega \rightarrow X$ be measurable. Then $T(\square, x(\square)): \Omega \rightarrow X$ is a $X$-valued measurable function.

Lemma 2.3[6]: Let $k \in C(\Omega \times I \times I \times X, X)$. Then for any $u \in C(\Omega \times I, X)$, the function $\int_{0}^{t} K(\square, t, s, u(\square, s)) d s$ is $C(I, X)$ valued measurable.

Lemma 2.4[6]: Let $x_{0} \in C(\Omega \times I, X)$ and let $k \in C(\Omega \times I \times I \times X, X)$. Then the random nonlinear integral operator defined by

$$
T(\omega, x(\omega, t))=x_{0}(\omega, t)+\int_{t_{0}}^{t} k(\omega, t, s, x(\omega, t)) d s
$$

satisfies the following conditions:

(i) for each $x \in C(\Omega \times I, X), T(\square, x(\square, t))$ is a $C(I, X)$-valued measurable.

(ii) for each $\omega \in \Omega, T(\omega, \square): C(\Omega \times I, X) \rightarrow C(\Omega \times I, X)$ is continuous under the norm \|\|$_{I}$, whenever for all $(t, s, x) \in I \times I \times X,\|k(\omega, t, s, x)\| \leq M(\omega)$ where $M: \Omega \rightarrow(0, \infty)$ is a given function.

Let $(X, d)$ be a complete metric space and $T: X \rightarrow X$ be a self map. For each $x \in X$, let $O(T, x)=\left\{T^{n}(x): n \geq 0\right\} \quad$ denotes the orbit of $\quad T \quad$ at $\quad x \quad$ and for any $B \subset X, D_{d}(B)=\sup \{d(x, y): x, y \in B\}$ denotes the diameter of $B$.

Lemma 2.5[6]: Let $T$ be a continuous self mapping. Suppose that for each $x \in X, D_{d}(O(T, x))<\infty$. If there exist positive integers $p, q$ and a real number $\beta \in(0,1)$ such that for all $x, y \in X$,

$$
d\left(T^{p} x, T^{q} y\right) \leq \beta D_{d}(O(T, x) \cup O(T, y)) \text { holds. }
$$

Then for each $x \in X,\left\{T^{n} x\right\}_{n \geq 0}$ converges to a unique fixed point $x^{*}$ of $T$.

The random nonlinear Volterra integral equation under consideration has the form:

$$
x(\omega, t)=x_{0}(\omega, t)+\int_{t_{0}}^{t} k(\omega, t, s, x(\omega, s)) d s
$$

where $x_{0} \in C(\Omega \times I, X)$ and $k \in C(\Omega \times I \times I \times X, X)$.

A function $x: \Omega \times I \rightarrow X$ is said to be a global random solution of the random equation (I), if $x(\omega, t)$ satisfies the random equation (I) and $x \in C(\Omega \times I, X)$.

Theorem 2.1: Let $x_{0} \in C(\Omega \times I, X)$ and $k \in C(\Omega \times I \times I \times X, X)$; and let $T: \Omega \times C(\Omega \times I, X) \rightarrow X$ as in Lemma 2.4.Suppose following conditions are satisfied:

(A1): for each $\omega \in \Omega$ and for all $(t, s, x) \in I \times I \times X,\|k(\omega, t, s, x)\| \leq M(\omega)$ where $M: \Omega \rightarrow(0, \infty)$ is a given function. 
(A2): there exist positive integers $p, q$ and functions $L_{1}(\omega), L_{2}(\omega)$ from $\Omega$ to $(0, \infty)$ such that for each $\omega \in \Omega$ and for all $(t, s) \in I \times I$ and $x(\omega, s), y(\omega, s) \in C(\Omega \times I, X)$ the following inequality holds $\left\|k\left(\omega, t, s, T^{p-1}(\omega, x(\omega, s))\right)-k\left(\omega, t, s, T^{q-1}(\omega, y(\omega, s))\right)\right\|_{I}$ $\leq L_{1}(\omega) D_{\|\square\|_{I}} O(T, x(\omega, s))+L_{2}(\omega) D_{\|\square\|_{I}} O(T, y(\omega, s))$. where $T^{n}(\omega, x(\omega, t))=x_{0}(\omega, t)+\int_{t_{0}}^{t} k\left(\omega, t, s, T^{n-1}(\omega, x(\omega, s)) d s, n=1,2, \ldots\right.$ and $T^{0}(\omega, x(\omega, t))=x(\omega, t)$.

Then the random equation

$$
x(\omega, t)=x_{0}(\omega, t)+\int_{t_{0}}^{t} k(\omega, t, s, x(\omega, s)) d s
$$

where $x_{0} \in C(\Omega \times I, X)$ and $k \in C(\Omega \times I \times I \times X, X)$ has a unique global random solution $x^{*} \in C(\Omega \times I, X)$ and for each $x \in C(\Omega \times I, X)$, the sequence $\left\{T^{n}(\omega, x(\omega, t))\right\}_{n \geq 0}$ converges to $x^{*}(\omega, t)$ under the norm \|\|$_{I}$ for each $\omega \in \Omega$.

Proof: Clearly for each fixed $\omega \in \Omega,\left(C(\Omega \times I, X),\|\|_{I}\right)$ is also a separable Banach space. For a fixed $\omega \in \Omega$, we shall introduce a new norm on $C(\Omega \times I, X)$ by

Now,

$$
\|x(\omega, t)\|_{*}=\max _{t \in I} e^{-\left(L_{1}(\omega)+L_{2}(\omega)\right) t} \cdot\|x(\omega, t)\|_{I}
$$

and hence \|\|$_{*}$ and \|\|$_{I}$ are equivalent norms. Now we shall discuss the random equation (1).

By (A1) and Lemma 2.4, the random nonlinear integral operator

$T(\omega, \square):\left[C(\Omega \times I, X),\|\|_{I}\right] \rightarrow\left[C(\Omega \times I, X),\|\|_{I}\right]$ is continuous and so

$T(\omega, \square):\left[C(\Omega \times I, X),\|\|_{*}\right] \rightarrow\left[C(\Omega \times I, X),\|\|_{*}\right]$ is also continuous. From assumption (A2), it

follows that for each $\omega \in \Omega$,

$$
\begin{aligned}
& \left\|T^{p}(\omega, x(\omega, t))-T^{q}(\omega, y(\omega, t))\right\|_{*} \\
& =\max _{t \in I} e^{-\left(L_{1}(\omega)+L_{2}(\omega)\right) t} \cdot\left\|\int_{t_{0}}^{t}\left[k\left(\omega, t, s, T^{p-1}(\omega, x(\omega, s))\right)-k\left(\omega, t, s, T^{q-1}(\omega, y(\omega, s))\right) d s\right]\right\|_{I} \\
& \leq \max _{t \in I} \int_{t_{0}}^{t} \int_{e}^{\left(L_{1}(\omega)+L_{2}(\omega)\right)(s-t)-\left(L_{1}(\omega)+L_{2}(\omega)\right) s}\left\|\left[k\left(\omega, t, s, T^{p-1}(\omega, x(\omega, s))\right)-k\left(\omega, t, s, T^{q-1}(\omega, y(\omega, s))\right)\right]\right\|_{I} d s \\
& \leq \max _{t \in I} \int_{t_{0}}^{t}{ }_{e}^{\left(L_{1}(\omega)+L_{2}(\omega)\right)(s-t)} d s \cdot\left\{L_{1}(\omega) D_{\|\| \|_{*}} O(T, x(\omega, s))+L_{2}(\omega) D_{\|\| \|_{*}} O(T, y(\omega, s))\right\}
\end{aligned}
$$

and by routine calculation,

$$
\begin{aligned}
& \leq \frac{1}{L_{1}(\omega)+L_{2}(\omega)} \cdot\left[1-e^{-\left(L_{1}(\omega)+L_{2}(\omega)\right) a}\right] \cdot\left\{\left\{L_{1}(\omega)+L_{2}(\omega)\right\} D_{\|\|_{*}} O(T, x(\omega, s))\right. \\
& \left.+\left\{L_{1}(\omega)+L_{2}(\omega)\right\} D_{\|\| \|_{*}} O(T, y(\omega, s))\right\} \\
& \leq \beta .\left\{D_{\|\| \|_{*}} O(T, x(\omega, s))+D_{\|\|_{*}} O(T, x(\omega, s))\right\}
\end{aligned}
$$


where $\beta=1-e^{-\left(L_{1}(\omega)+L_{2}(\omega)\right) a}<1$ for each $\omega \in \Omega$.

Since for each $\omega \in \Omega$ and for all $(t, s, x) \in I \times I \times X$, we have $\|k(\omega, t, s, x)\| \leq M(\omega)$, it is easy to see that for each $\omega \in \Omega$ and for each $x \in C(\Omega \times I, X), D_{\|\| \|_{*}} O(T, x(\omega, s))<\infty$.

Then from Lemma 2.5 it follows that there exists a unique function $x^{*}: \Omega \times I \rightarrow X$ such that for each fixed $\omega \in \Omega, x^{*}(\omega, t)$ satisfies equation (1) and for each $x \in C(\Omega \times I, X)$ the sequence $\left\{T^{n}(\omega, x(\omega, t))\right\}_{n \geq 0}$ converges to $x^{*}(\omega, t)$ under the norm \|\|$_{*}$ and so $\left\{T^{n}(\omega, x(\omega, t))\right\}_{n \geq 0}$ converges to $x^{*}(\omega, t)$ under the norm \|\|$_{I}$.

On the other hand, from Lemma 2.4, it follows that $\left\{T^{n}(\omega, x(\omega, t))\right\}_{n \geq 0}$ is a sequence of $C(I, X)$ measurable function. Therefore Lemma 2.1 applies to conclude that $x^{*}(\omega, t) \in C(\Omega \times I, X)$. Thus $x^{*}(\omega, t)$ is a unique global random solution of Volterra Integral Equation as in (1).

Corollary 2.1: Putting $L_{1}(\omega)=L_{2}(\omega)=L(\omega)$ (say), Theorem 3.1 [6] follows.

Corollary 2.2: Let $x_{0} \in C(\Omega \times I, X)$ and $k \in C(\Omega \times I \times I \times X, X)$ and suppose following conditions are satisfied

(i) condition (A1) of theorem 2.1 holds,

(ii) there exists function $L(\omega): \Omega \rightarrow(0, \infty)$ such that for each $\omega \in \Omega$ and for all $(t, s) \in I \times I$, $x, y \in C(\Omega \times I, X)$ the following inequality holds.

$$
\|k(\omega, t, s, x(\omega, s))-k(\omega, t, s, y(\omega, s))\|_{I} \leq L(\omega)\|x(\omega, s)-y(\omega, s)\|_{I} .
$$

Then for $x(\omega, t) \in C(\Omega \times I, X)$ the sequence $\left\{T^{n}(\omega, x(\omega, t))\right\}$ converges to a unique global random solution $x^{*}(\omega, t)$ of the random equation (1) under the norm \|\|$_{I}$.

Proof: Clearly Corollary 2.2 is a special case of Theorem 2.1 with $p=q=1$. Before we close we cite an example in support of Theorem 2.1 as proved above.

Example 2.1: In support of main theorem, following is an example

Take $\Omega=R, X=R, I=[0,1]$ with usual topology of reals. Define $T: \Omega \times C(\Omega \times I, X) \rightarrow X$ to read as $T(\omega, x(\omega, t))=x(\omega, 0)+\int_{0}^{t} \alpha \cdot e^{\alpha s} d s$, where $0<\alpha<1$. Then routine check up shows that $T$ fits in Main theorem and on actual computation, we find the unique solution as $x(\omega, t)=e^{\alpha t}$ for all $\omega \in \square(\Omega)$.

\section{Acknowledgement:}

The first author is being supported by University Grants Commission's (Regional office, Kolkata) Minor Research Project (letter no F.FSW-032/11-12 (ERO), dated 08.08.2011) for this work.

\section{References:}

[1]. A. Spacek, Zufallige Gleichungen, Czechoslovak Mathematical Journal, 5(80), 1955, 462-466.

[2]. A. C. H. Lee and W.J.Padgett, Random Contractors with random nonlinear majorant functions, Nonlinear Analysis, TMA, 3, 1979, 707-715.

[3]. A. T. Bharucha-Reid, Random Integral Equations, Acad. Press New York, 1972.

[4]. C. P. Tsokos, On a stochastic integral equation of the volterra type, Math. Systems Theory, 3, 1969, $222-231$. 
[5]. Ding Xieping and Wang Fan, Solutions for a system of nonlinear random integral and differential equation under weak topology, Applied Mathematics and Mechanics, Su, Shanghai, China, 18(8), 1997,721-737.

[6]. Ding Xieping, Criterion for the existence of solutions to random integral and differential equations, Applied Mathematics and Mechanics,6(3),1985,269-275

[7]. H.W. Engl, A general stochastic fixed point theorem for continuous random operators on stochastic domaine, J. Math. Anal Appl., 66(1), 1978, 220-231.

[8]. H. W. Anderson, Stochastic Integral Equations, Ph.D. Dissertation, University of Tennesse, Nashville, Tenn., 1966.

[9]. M.Saha and L.Debnath, Random fixed point of mapping over a Hilbert space with probability measure, Adv.Stud.Contemp. Math., 18(1), 2009.97-104.

[10]. M.Saha, M.Gangopadhyay and Debnath, Random fixed point theorems in a separable Banach space with probability Measure, Int J.Contemp.Math.Sci., 5, 2010, 1115-1129.

[11]. O. Hans, Reduzierendae Zufallige Transformationen, Czechoslovak Math. J. 7(82), 1957, 154-158.

[12]. R. Subramonium and K. Balachandram, Existence of solutions of a class of stochastic volterra Integral Equations with application to chemology, J. Austral. Math. Soc. Ser. B 41 (1999), 93-104.

[13]. S. Itoh, Random fixed point theorems with application to random differential equations in Banach spaces, J. Math. Anal. Appl. 67,1979, 261-273.

[14]. W. J. Padgett and C. P. Tsokos, Random Integral Equation with application to stochastic system, Lecture Notes in Mathematics, 233, Springer-Verlog, Berlin, 1971. 